Investigaciones geográficas, $n .^{\circ} 45$ (2008) pp. 141-162 ISSN: 0213-4691
Instituto Universitario de Geografía Universidad de Alicante

\title{
EVALUACIÓN DE LA APTITUD CLIMÁTICO- TURÍSTICA PARA EL TURISMO DE SOL Y PLAYA EN ALICANTE (1974-2003)
}

\author{
Emilio Martínez Ibarra \\ Instituto Universitario de Geografía \\ Universidad de Alicante
}

\section{Características de los tipo de tiempo considerados}

Con la finalidad de evaluar el potencial climático para la práctica del turismo de sol y playa en Alicante se hará uso de la metodología de tipos de tiempo ${ }^{1}$ propuesta por Martínez (2008), la cual está basada en el análisis de la relación entre la densidad de uso de la playa de Levante de Benidorm (práctica del bronceado y baño) y las principales variables meteorológicas en materia turística (temperatura máxima, fracción de radiación solar, precipitación, humedad relativa y velocidad del viento) ${ }^{2}$. Amén, fundamentalmente, la metodología se apoya en las consideraciones realizadas por Besancenot, Mounier y De Lavenne (1978); Besancenot (1985; 1991); Gómez (2000); Batista y Matos (2004); De Freitas, Scout y McBoyle (2004). Asimismo, se han analizado las nuevas aportaciones de Scout, Gössling y De Freitas (2007); Andrade, Aljofarado y Oliveira (2007); y Matzarakis (2007).

Tal y como se observa en la tabla 1 , el método se caracteriza por la diferenciación de trece clases, ordenadas de acuerdo a su grado de potencialidad. Básicamente, sus características son las que a continuación se detallan:

1. Este procedimiento para evaluar el potencial climático-turístico resulta el más adecuado según numerosos autores (vid. Besancenot, Mounier y De Lavenne, 1978; Cuadrat, 1981; Besancenot, 1991; Gómez, 2003; o Andrade, Aljofarado y Oliveira, 2007).

2. Vid. Besancenot (1991) y de C.R. Freitas (1990; 2001; 2003 y 2005). 


\section{Tipo 1: Tiempo muy bueno soleado}

Este tipo viene definido por una fracción de insolación o radiación de al menos un $75 \%$ u $80 \%$, respectivamente, la inexistencia de precipitación, una temperatura cálida, comprendida entre los $28^{\circ} \mathrm{C}$ y los $31^{\circ} \mathrm{C}$, y un confort aceptable (con valores del poder refrigerante del aire de al menos $58 \mathrm{~W} / \mathrm{m}^{2}$ y un índice termo-higrométrico que no supera los $26,5^{\circ} \mathrm{C}$ ), una tensión parcial de vapor de agua comprendida entre los 4 y los $25 \mathrm{hPa}$, y viento poco importante, con velocidad inferior a $\operatorname{los} 8 \mathrm{~m} / \mathrm{s}$.

En suma, tiempo cálido y soleado ideal para el disfrute de la práctica turística analizada. Contexto atmosférico en el que el organismo no deberá realizar grandes esfuerzos para mantener sus constantes térmicas e hídricas.

Por todo ello, se infiere que haya sido catalogado como la mejor clase de tiempo para el turismo de sol y playa.

\section{Tipo 2: Tiempo bastante bueno soleado}

Este escenario meteorológico viene caracterizado por una fracción de insolación o radiación de al menos un $75 \%$ u $80 \%$, respectivamente, la inexistencia de precipitación, una temperatura algo cálida, de $25^{\circ} \mathrm{C}$ a $28^{\circ} \mathrm{C}$, una situación de confort adecuada a la sombra (con valores del poder refrigerante del aire de al menos $58 \mathrm{~W} / \mathrm{m}^{2}$ y un índice termo-higrométrico que no supera los $26,5^{\circ} \mathrm{C}$ ) y una tensión parcial de vapor de agua comprendida entre los 4 y los $25 \mathrm{hPa}$. Además, el viento es poco importante, con una velocidad inferior a los $8 \mathrm{~m} / \mathrm{s}$.

En definitiva, tiempo relativamente cálido y soleado bastante apropiado para el disfrute del sol y la playa.

Así pues, atendiendo a su grado de aptitud, ha sido definido como el segundo tipo de tiempo para la práctica recreativa aquí analizada.

\section{Tipo 3: Tiempo cálido y pesado}

Respecto a los tipos que le preceden, su principal particularidad no es otra sino unas condiciones de confort térmico o higrométrico que se aproximan a los límites superiores estimados como aceptables.

En efecto, además de venir definido por una fracción de insolación o radiación de al menos un $75 \%$ u $80 \%$, respectivamente, la inexistencia de precipitación y viento de escasa importancia, inferior a los $8 \mathrm{~m} / \mathrm{s}$, queda determinado por una temperatura bastante cálida, comprendida entre los $31-33^{\circ} \mathrm{C}$ y los $33-35^{\circ} \mathrm{C}$, según los casos. 
Tabla 1. Tipos de tiempo definidos como adecuados para la práctica del turismo de sol y playa en el litoral alicantino

\begin{tabular}{|c|c|c|c|}
\hline $\begin{array}{c}\text { Tipo } 1 \text {. Tiempo muy } \\
\text { bueno soleado } \\
\mathrm{Fi} \geq 75 \% \text { o } \mathrm{Fr} \geq 80 \% \\
\mathrm{D}=0 \mathrm{~h} \text { o } \mathrm{P}=0 \mathrm{~mm} \\
28 \leq \mathrm{Tx}<31^{\circ} \mathrm{C} \\
\mathrm{K} \geq 58 \mathrm{~W} / \mathrm{m}^{2} \\
\mathrm{THI} \leq 26,5^{\circ} \mathrm{C} \\
\mathrm{V}<8 \mathrm{~m} / \mathrm{s} \\
4<\mathrm{U}<25 \mathrm{hPa}\end{array}$ & $\begin{array}{c}\text { Tipo } 2 \text {. Tiempo } \\
\text { bastante bueno } \\
\text { soleado } \\
\mathrm{Fi} \geq 75 \% \text { h o } \mathrm{Fr} \geq 80 \% \\
\mathrm{D}=0 \mathrm{~h} \text { o } \mathrm{P}=0 \mathrm{~mm} \\
25 \leq \mathrm{Tx}<28^{\circ} \mathrm{C} \\
\mathrm{K} \geq 58 \mathrm{~W} / \mathrm{m}^{2} \\
\mathrm{THI} \leq 26,5^{\circ} \mathrm{C} \\
\mathrm{V}<8 \mathrm{~m} / \mathrm{s} \\
4<\mathrm{U}<25 \mathrm{hPa}\end{array}$ & $\begin{array}{c}\text { Tipo } 3 . \text { Tiempo cálido } \\
\text { y pesado } \\
\text { Fi } \geq 75 \% \mathrm{~h} \text { o } \mathrm{Fr} \geq 80 \% \\
\mathrm{D}=0 \mathrm{~h} \text { o } \mathrm{P}=0 \mathrm{~mm} \\
\mathrm{THI} \leq 28,5 \\
\mathrm{~V}<8 \mathrm{~m} / \mathrm{s} \\
\mathrm{U}<31,3 \mathrm{hPa} \\
31 \leq \mathrm{Tx}<33^{\circ} \mathrm{C} \\
\text { y/o } 0 \leq \mathrm{K}<58 \mathrm{~W} / \mathrm{m}^{2} \\
\text { y/o } 26,5<\mathrm{THI} \leq 28,5^{\circ} \mathrm{C} \\
\text { y/o } 25 \leq \mathrm{U}<31,3 \mathrm{hPa} \\
\mathrm{o} \\
33 \leq \mathrm{Tx}<35^{\circ} \mathrm{C} \\
\mathrm{THI} \leq 26,5^{\circ} \mathrm{C}\end{array}$ & $\begin{array}{c}\text { Tipo } 4 . \text { Tiempo bueno } \\
\text { soleado } \\
\text { Fi } \geq 75 \% \text { h o Fr } \geq 80 \% \\
\mathrm{D}=0 \mathrm{~h} \text { o } \mathrm{P}=0 \mathrm{~mm} \\
23 \leq \mathrm{Tx}<25^{\circ} \mathrm{C} \\
\mathrm{K} \geq 58 \mathrm{~W} / \mathrm{m}^{2} \\
\mathrm{THI} \leq 26,5^{\circ} \mathrm{C} \\
\mathrm{V}<8 \mathrm{~m} / \mathrm{s} \\
4<\mathrm{U}<25 \mathrm{hPa}\end{array}$ \\
\hline $\begin{array}{c}\text { Tipo } 5 \text {. Tiempo } \\
\text { aceptable con } \\
\text { cobertura nubosa } \\
\text { parcial } \\
\text { 35\% } \leq \mathrm{Fi}<75 \% \text { o } 50 \% \\
\leq \mathrm{Fr}<80 \% \\
\mathrm{D}=0 \mathrm{~h} \text { o } \mathrm{P}=0 \mathrm{~mm} \\
\mathrm{THI} \leq 28,5^{\circ} \mathrm{C} \\
\mathrm{V}<8 \mathrm{~m} / \mathrm{s} \\
4<\mathrm{U}<31,3 \mathrm{hPa} \\
25 \leq \mathrm{Tx}<33^{\circ} \mathrm{C} \\
\mathrm{K} \geq 0 \mathrm{~W} / \mathrm{m}^{2} \\
\quad \mathrm{O} \\
33 \leq \mathrm{Tx}<35^{\circ} \mathrm{C} \\
\mathrm{THI} \leq 26,5^{\circ} \mathrm{C}\end{array}$ & $\begin{array}{c}\text { Tipo } 6 . \text { Tiempo } \\
\text { aceptable algo fresco } \\
\text { soleado } \\
\text { Fi } \geq 75 \% \text { h o Fr } \geq 80 \% \\
\mathrm{D}=0 \mathrm{~h} \mathrm{o} \mathrm{P}=0 \mathrm{~mm} \\
20 \leq \mathrm{Tx}<23^{\circ} \mathrm{C} \\
\mathrm{K} \geq 58 \mathrm{~W} / \mathrm{m}^{2} \\
\mathrm{THI} \leq 26,5^{\circ} \mathrm{C} \\
\mathrm{V}<8 \mathrm{~m} / \mathrm{s} \\
4<\mathrm{U}<25 \mathrm{hPa}\end{array}$ & $\begin{array}{c}\text { Tipo } 7 \text {. Tiempo } \\
\text { aceptable con breve } \\
\text { episodio lluvioso } \\
\text { Fi } \geq 50 \% \text { o } \mathrm{Fr} \geq 66 \% \\
0,1 \leq \mathrm{P}<1 \mathrm{~mm} \\
\mathrm{THI} \leq 28.5^{\circ} \mathrm{C} \\
\mathrm{V}<8 \mathrm{~m} / \mathrm{s} \\
4<\mathrm{U}<31,3 \mathrm{hPa} \\
25 \leq \mathrm{Tx}<33^{\circ} \mathrm{C} \\
\mathrm{K} \geq 0 \mathrm{~W} / \mathrm{m}^{2} \\
\mathrm{O} \\
33 \leq \mathrm{Tx}<35^{\circ} \mathrm{C} \\
\mathrm{THI} \leq 26,5^{\circ} \mathrm{C}\end{array}$ & $\begin{array}{c}\text { Tipo } 8 \text {. Tiempo } \\
\text { aceptable con } \\
\text { episodio lluvioso } \\
\text { Fi }>50 \% \text { o Fr }>66 \% \\
1 \leq \mathrm{P}<5 \mathrm{~mm} . \\
\mathrm{THI} \leq 28.5^{\circ} \mathrm{C} \\
\mathrm{V}<8 \mathrm{~m} / \mathrm{s} \\
4<\mathrm{U}<31,3 \mathrm{hPa} \\
25 \leq \mathrm{Tx}<33^{\circ} \mathrm{C} \\
\mathrm{K} \geq 0 \mathrm{~W} / \mathrm{m} \\
\mathrm{O} \\
33 \leq \mathrm{Tx}<35^{\circ} \mathrm{C} \\
\mathrm{THI} \leq 26,5^{\circ} \mathrm{C}\end{array}$ \\
\hline 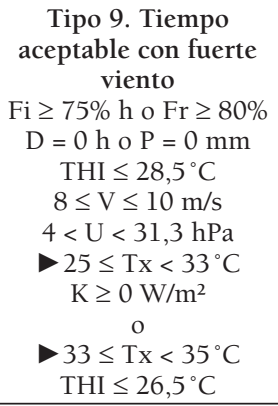 & $\begin{array}{c}\text { Tipo } 10 \text {. Tiempo } \\
\text { aceptable fresco } \\
\text { soleado } \\
\mathrm{Fi} \geq 75 \% \mathrm{~h} \text { o } \mathrm{Fr} \geq 80 \% \\
\mathrm{D}=0 \mathrm{~h} \text { o } \mathrm{P}=0 \mathrm{~mm} \\
18 \leq \mathrm{Tx}<20^{\circ} \mathrm{C} \\
\mathrm{THI} \leq 26,5^{\circ} \mathrm{C} \\
\mathrm{K} \geq 58 \mathrm{~W} / \mathrm{m}^{2} \\
\mathrm{~V}<8 \mathrm{~m} / \mathrm{s} \\
4<\mathrm{U}<25 \mathrm{hPa}\end{array}$ & $\begin{array}{c}\text { Tipo } 11 \text {. Tiempo } \\
\text { aceptable relativamente } \\
\text { fresco con cobertura } \\
\text { nubosa parcial } \\
35 \% \leq \mathrm{Fi}<75 \% \text { o } 50 \% \leq \\
\mathrm{Fr}<80 \% \\
\mathrm{D}=0 \mathrm{~h} \text { o } \mathrm{P}=0 \mathrm{~mm} \\
23 \leq \mathrm{Tx}<25^{\circ} \mathrm{C} \\
\mathrm{K} \geq 58 \mathrm{~W} / \mathrm{m}^{2} \\
\mathrm{THI} \leq 26,5^{\circ} \mathrm{C} \\
\mathrm{V}<8 \mathrm{~m} / \mathrm{s} \\
4<\mathrm{U}<25 \mathrm{hPa}\end{array}$ & $\begin{array}{c}\text { Tipo } 12 . \text { Tiempo } \\
\text { aceptable } \\
\text { relativamente fresco } \\
\text { con breve episodio } \\
\text { lluvioso } \\
\mathrm{Fi} \geq 50 \% \text { o Fr } \geq 66 \% \\
0,1 \leq \mathrm{P}<1 \mathrm{~mm} \\
23 \leq \mathrm{Tx}<25^{\circ} \mathrm{C} \\
\mathrm{K} \geq 58 \mathrm{~W} / \mathrm{m}^{2} \\
\mathrm{THI} \leq 26,5^{\circ} \mathrm{C} \\
\mathrm{V}<8 \mathrm{~m} / \mathrm{s} \\
4<\mathrm{U}<25 \mathrm{hPa}\end{array}$ \\
\hline \multicolumn{4}{|c|}{$\begin{array}{l}\text { Tipo 13. Tiempo aceptable relativamente fres } \\
\qquad \begin{array}{c}\mathrm{Fi} \geq 75 \% \mathrm{~h} \text { o } \mathrm{Fr} \geq 80 \% \\
\mathrm{D}=0 \mathrm{~h} \text { o } \mathrm{P}=0 \mathrm{~mm} \\
23 \leq \mathrm{T} x<25^{\circ} \mathrm{C} \\
\mathrm{K} \geq 58 \mathrm{~W} / \mathrm{m}^{2} \\
\mathrm{THI} \leq 26,5^{\circ} \mathrm{C} \\
8<\mathrm{V} \leq 10 \mathrm{~m} / \mathrm{s} \\
4<\mathrm{U}<25 \mathrm{hPa}\end{array}\end{array}$} \\
\hline
\end{tabular}

Fuente: Elaboración propia. 
Por tanto, situación que hará actuar de forma más o menos importante a los agentes que intervienen para mantener las constantes físicas y biológicas de nuestro organismo, generándose de este modo cierto estrés.

No obstante, el turista de sol y playa muestra cierta flexibilidad ante ambientes térmicos de este tipo, dada la facultad de estos estados de tiempo para el disfrute de la práctica más deseada por el segmento de consumidores objeto de análisis ${ }^{3}$.

De esta forma, por su idoneidad, este escenario de tiempo ha sido definido en tercer lugar.

\section{Tipo 4: Tiempo bueno soleado}

Estado de temperie determinada por una fracción de insolación o radiación de al menos un $75 \%$ u $80 \%$, respectivamente, la inexistencia de precipitación, una temperatura confortable a la sombra, entre los $23^{\circ} \mathrm{C}$ y los $25^{\circ} \mathrm{C}$, con valores del poder refrigerante del aire de al menos $58 \mathrm{~W} / \mathrm{m}^{2}$, un índice termo-higrométrico que no supera los $26,5^{\circ} \mathrm{C}$, una tensión parcial de vapor de agua concretada entre los 4 y los $25 \mathrm{hPa}$, y viento poco importante, con una velocidad que no alcanza la cifra de los $8 \mathrm{~m} / \mathrm{s}$.

Así pues, ambiente confortable y soleado que muestra aún bastantes aptitudes para el disfrute del sol y la playa, de ahí su catalogación en cuarto lugar.

\section{Tipo 5: Tiempo aceptable con cobertura nubosa parcial}

Este contexto atmosférico difiere del resto de los hasta aquí definidos por la presencia de nubosidad, con una fracción de insolación o radiación comprendida entre el $35 \%$ y el $75 \%$ o el 50 y el $80 \%$, respectivamente. Empero, no muestra precipitación, el viento es poco importante, inferior a $8 \mathrm{~m} / \mathrm{s}$, y la temperatura oscila entre los $25^{\circ} \mathrm{C}$ y $35^{\circ} \mathrm{C}$, según los valores alcanzados por el índice termo-higrométrico.

En conjunto, tiempo que, a pesar de presentar una cobertura nubosa parcial, sigue manteniendo ciertas aptitudes para el turismo de sol y playa. De hecho, al mostrar como máximo inconveniente la aparición de nubosidad, se ha clasificado en el quinto lugar.

3. A este respecto, FJ. Chávez (2002: 87-89) señala que, en ocasiones, se aceptan, e incluso se disfrutan, condiciones de «inconfort» térmico, como, por ejemplo, al broncearse en la playa. A las mismas conclusiones llegaron A. Dauphiné y N. Ghilardi (1978: 5). 


\section{Tipo 6: Tiempo aceptable algo fresco soleado}

Las características de este tipo son idénticas al resto de los ambientes definidos como soleados, con la única salvedad del umbral térmico, en este caso representado por una temperatura que puede abarcar de $20^{\circ} \mathrm{C}$ a $23^{\circ} \mathrm{C}$.

Así pues, tiempo confortable y soleado que muestra aún aptitudes para el disfrute del sol y la playa.

\section{Tipo 7: Tiempo aceptable con breve episodio lluvioso}

Una precipitación de escasa importancia, inferior al mm, en un ambiente relativamente cálido o cálido, con una temperatura comprendida entre los $25^{\circ} \mathrm{C}$ y $35^{\circ} \mathrm{C}$ (según los valores del índice termo-higrométrico), una fracción de insolación o radiación de al menos un $50 \%$ o $66 \%$, respectivamente, y viento poco importante, inferior a $8 \mathrm{~m} / \mathrm{s}$, son los atributos meteorológicos que particularizan este tipo de temperie.

Como quiera que el episodio lluvioso es de escasa significación, el ambiente térmico resulta cálido y el período sin nubosidad es importante, esta variedad de ambiente meteorológico ha sido estimada como aceptable, en séptimo lugar.

\section{Tipo 8: Tiempo aceptable con episodio lluvioso}

Únicamente se distingue del anterior por la cantidad de precipitación, pues ésta puede oscilar entre 1 y $5 \mathrm{~mm}$.

A pesar de estar ligado a una cantidad de lluvia relativamente importante, al combinarse esta circunstancia con unos valores de insolación y/o radiación relativamente elevados y un ambiente cálido, este contexto atmosférico se ha definido como adecuado, en octava posición.

\section{Tipo 9: Tiempo aceptable con fuerte viento}

Una velocidad del viento algo importante, comprendida entre 8 y $10 \mathrm{~m} / \mathrm{s}$, en un contexto relativamente cálido o cálido, con una temperatura que puede oscilar entre los $25^{\circ} \mathrm{C}$ y $35^{\circ} \mathrm{C}$, y una fracción de insolación o radiación de al menos un $75 \%$ u $80 \%$, según los casos, constituyen los umbrales claves que concretan este escenario, clasificado en noveno lugar.

\section{Tipo 10: Tiempo aceptable fresco soleado}

Las particularidades que definen a este «género» resultan idénticas al resto de las temperies clasificadas como soleadas, a excepción de la horquilla térmica, 
que restringe estos escenarios atmosféricos a aquellos ambientes determinados por valores de $18^{\circ} \mathrm{C}$ y $20^{\circ} \mathrm{C}$.

Así, tiempo que se haya en los límites de la confortabilidad térmica a la sombra en reposo, en el seno de un contexto soleado, que muestra aún ciertas aptitudes para el disfrute del sol y la playa.

Tipo 11: Tiempo aceptable relativamente fresco con cobertura nubosa parcial

A una fracción de insolación o radiación relativamente reducida, comprendida entre el $35 \%$ y el $75 \%$ o el $50 \%$ y el $80 \%$, se añade como inconveniente un escenario térmico suave, con valores entre los $23^{\circ} \mathrm{C}$ y $25^{\circ} \mathrm{C}$; si bien, el resto de sus características resultan ideales. Serie de pros y contras que han determinado que esta «variedad» haya sido colocada, de acuerdo con su aptitud climático-turística, en undécimo lugar.

Tipo 12: Tiempo aceptable relativamente fresco con breve episodio lluvioso

$\mathrm{Al}$ inconveniente de la posible presencia de nubosidad, bien es cierto que con fracciones de insolación o radiación de al menos un 50\% o 66\%, respectivamente, se une un obstáculo mayor, esto es, el desencadenamiento de un breve episodio lluvioso, con cantidades inferiores a $1 \mathrm{~mm}$. Amén, el marco térmico tampoco resulta el más adecuado para la práctica del turismo de sol y playa, con valores comprendidos entre los $23^{\circ} \mathrm{C}$ y $25^{\circ} \mathrm{C}$.

Por todo ello, se ha optado por encasillar a este estado de situación atmosférica en penúltimo lugar.

\section{Tipo 13: Tiempo aceptable relativamente fresco con fuerte viento}

Los principales escollos que impregnan a esta situación atmosférica son: de un lado, un viento relativamente importante, comprendido entre los 8 y 10 $\mathrm{m} / \mathrm{s} ; \mathrm{y}$, de otro, un contexto térmico suave. El resto de sus propiedades meteorológicas resultan ideales.

Como la presencia del viento se entiende más o menos continua, se ha optado por clasificar a este «género» en último lugar. 


\section{Valoración inicial del potencial climático-turístico para el turismo de sol y playa en Alicante}

Comentadas las características fundamentales de los distintos tipos de tiempo manejados, cabe una primera aproximación relativa a la evaluación del potencial climático-turístico para el turismo de sol y playa en Alicante $e^{4}$.

En este sentido, en primer lugar, se han distinguido, sin más, situaciones aceptables y desfavorables para la práctica recreativa objeto de estudio. A este respecto, se observa un ligero predominio de las primeras, con cifras porcentuales, según el total del período analizado, de $55,73 \%$ y $44,27 \%$, respectivamente (tabla 2).

De otro lado, se ha valorado la impronta media de cada tipo de tiempo definido como aceptable para el turismo de sol y playa. Por lo que a ello atañe, se ha observado un dominio de los tipos 2, 6, 3 y 1 , con valores porcentuales de $8,52 \%, 8,41 \%, 7,90 \%$ y $7,80 \%$, en cada caso (tabla 2 ). Próximos a éstos han quedado las clases 5,10 y 4 , y ya a una distancia importante las variantes 11 , $7,9,8,12$ y 13 , especialmente estas cinco últimas, con frecuencias de 2,15\%, $1,72 \%, 0,65 \%, 0,56 \%$ y $0,16 \%$, respectivamente (tabla 2 ).

Así pues, entre los tipos de tiempo favorables para la práctica turística, tal y como se observa en la tabla 2, han dominado ampliamente los ambientes soleados, sin precipitación y sin viento molesto, tipos $2,6,3,1,10$ y 4, con el $78 \%$ de los casos. Con respecto a ello, resulta necesario indicar que han predominado los tipos cálidos $(1,2$ y 3$)$ frente a los confortables y algo frescos (4, 6 y 10), con el $43 \%$ y el $35 \%$, respectivamente. Además, dentro de este grupo de tipos dominantes despunta la clase de tiempo cuyo inconveniente principal estriba en su grado de cobertura nubosa (tipo 5), con el 6,74\% del total de las jornadas analizadas. Ya a una distancia considerable, han aparecido los ambientes confortables con cobertura nubosa parcial (tipo 11) y el primer subtipo definido por la presencia de precipitación (7), con el 2,15\% y el $1,72 \%$, respectivamente. Las variedades ligadas a la existencia de un viento relativamente inconfortable (tipos 7 y 9), así como aquellas otras con caída de precipitación (tipos 8 y 12) completan la serie de las temperies aceptables más infrecuentes, con el $1,72 \%, 0,65 \%, 0,56 \%$ y $0,26 \%$, en cada caso.

De este modo, aparecen elevadas frecuencias de tipos aptos para la práctica recreativa aquí objeto de estudio. Ello responde a la posición de abrigo aerológico y orográfico del sector analizado.

4. Los datos analizados en la presente investigación se corresponden con los recogidos por el Agencia Estatal de Meteorología (AEMET) en el observatorio Ciudad Jardín de la ciudad de Alicante, durante el período 1973-2004. 
Por último, conviene remarcar la distribución acampanada, ligeramente achatada durante el centro del estío, de las frecuencias de los tipos de tiempo clasificados como favorables. Dicha forma del reparto porcentual encuentra razón de ser en la coincidencia de factores diversos, si bien íntimamente relacionados. Éstos son: un desplazamiento en latitud de los Centros de Acción en estío, de acuerdo con el Mecanismo Cósmico de las Estaciones; un aumento de la manifestación de las invasiones de aire cálido; y un incremento en la altura del sol, con la consiguiente acentuación de los valores de radiación solar y su eficacia. Todos estos factores coadyuvan para determinar registros térmicos elevados, en el seno de condiciones atmosféricas mayoritariamente estables, con elevadas fracciones de insolación. En definitiva, temperies ideales para el desarrollo del turismo de sol y playa. En cambio, durante los equinoccios y, particularmente, con ocasión del periodo invernal los tipos de tiempo adecuados dejan paso al predominio de aquellos contextos meteorológicos definidos como desfavorables (Tabla 2).

\section{Análisis de frecuencias de los tipos de tiempo definidos como aceptables en Alicante}

\section{Tipo 1: Tiempo muy bueno soleado}

La frecuencia media anual de este tipo de tiempo durante el periodo analizado en el observatorio de Alicante (Ciudad Jardín) presenta una especial vocación por el periodo cálido del año, con porcentajes de al menos un 14\% desde la segunda decena de junio hasta la tercera de septiembre.

Dicha predilección por la época más cálida se ve en cierta forma troncada durante el periodo central del estío, en coincidencia con el pico del género 3. Ello responde a la «degradación» de las condiciones de confort térmico durante la canícula estival, sobre la base de una elevada carga higrométrica del aire y de unas temperaturas altas. Así, durante el mes de agosto aparece un seno poco acentuado, con valores porcentuales comprendidos entre el $17,58 \%$ y el $14,67 \%$. En efecto, durante este periodo las brisas marinas pierden eficacia, de acuerdo con unas aguas mediterráneas cálidas, que inhiben, en buena medida, el poder refrigerante de los antedichos vientos de carácter local (Tabla 2).

\section{Tipo 2: Tiempo bastante bueno soleado}

Este tipo de tiempo muestra una distribución anual bimodal más acusada que la del tipo 1, pues las condiciones de confort térmico son más exigentes. Esto último determina que durante el periodo previo y posterior al estío esta clase 


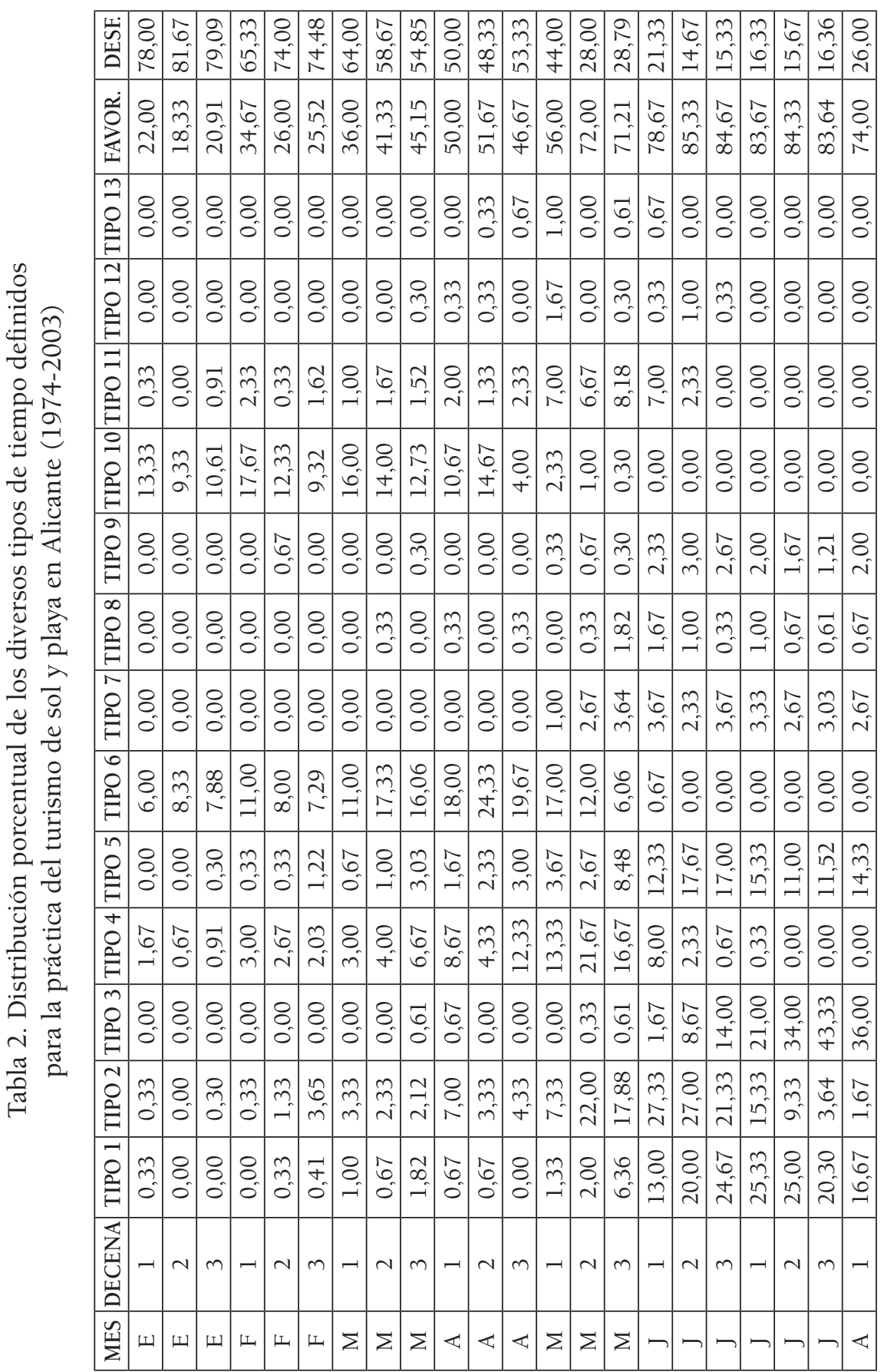




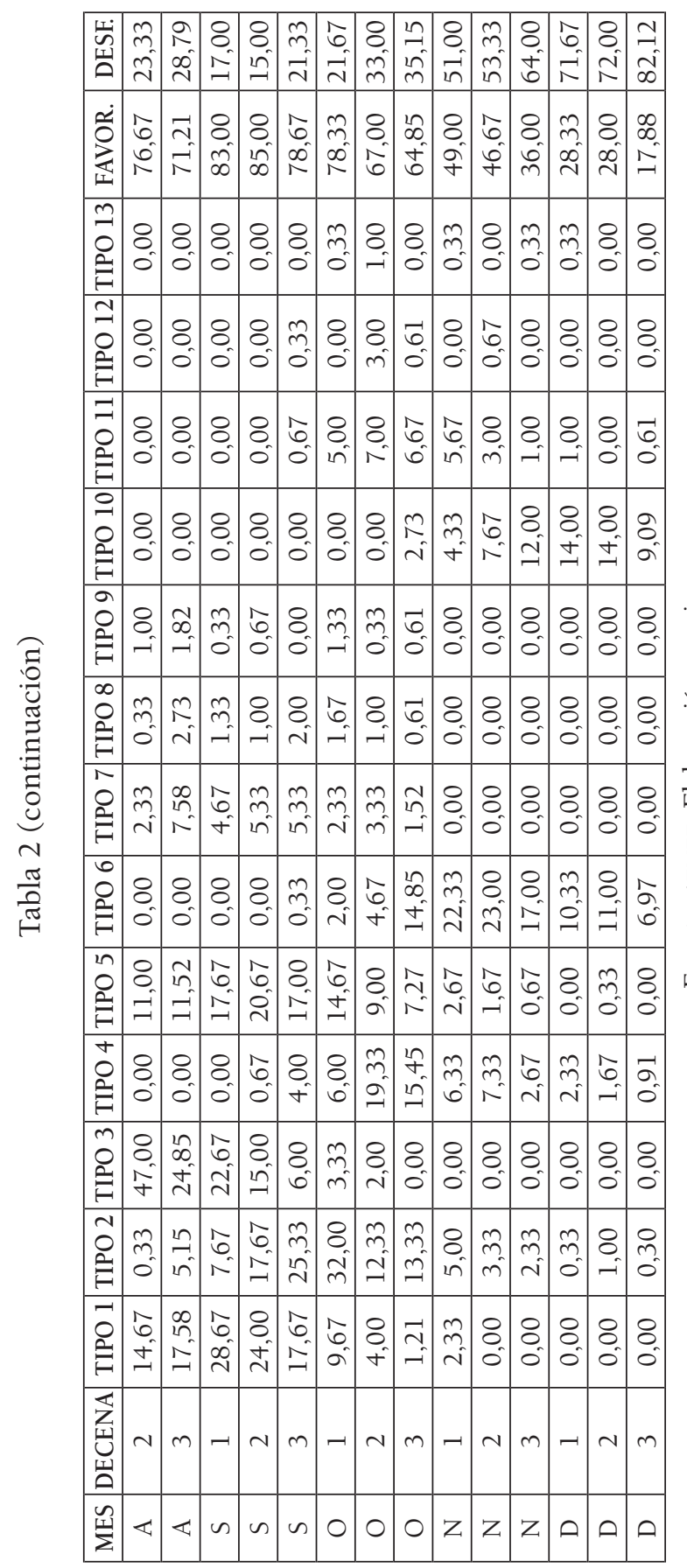

Investigaciones geográficas, n. ${ }^{\circ} 45$ (2008) 
prime sobre la primera. Por tanto, los valores porcentuales se sitúan por encima del tipo 1 hasta la segunda decena del mes de junio y a partir de la tercera decena del mes de septiembre; con la única salvedad de la primera y segunda decena del mes de enero, cuando las frecuencias de las dos clases, 1 y 2 , se han mostrado idénticas (tabla 2).

\section{Tipo 3: Tiempo cálido y pesado}

El tipo 3 aparece como el estado de temperie hegemónico desde la segunda decena del mes de julio hasta la tercera del mes de agosto, con frecuencias para dicho margen temporal que superan el 24\%. Los máximos decenales alcanzan cifras superiores al $40 \%$, con valores del $43,33 \%$ y $47 \%$ en la tercera decena del mes de julio y la segunda de agosto; preponderancia no registrada por ningún otro tipo en alguna otra decena (tabla 2).

Cabe asimismo indicar que el pico máximo de este tipo muestra un cierto retraso con respecto a la mayor cantidad de radiación solar recibida, dado el papel regulador que ejerce el mar Mediterráneo y, en última instancia, las brisas, pues éstas transportan sus propiedades térmico-higrométricas (las del mar) hacia el espacio litoral terrestre.

\section{Tipo 4: Tiempo bueno soleado}

La mayor exigencia de confort térmico de esta temperie (respecto a los tipos 1 y 2) acentúa el carácter bimodal de su distribución de frecuencias anual, al tiempo que traslada sus máximos al centro de los equinoccios. En efecto, los valores más elevados se han registrado durante la segunda y tercera decena del mes de marzo y en la segunda y tercera de octubre. Los porcentajes alcanzados han sido de $21,67 \%, 16,67 \%, 19,33 \%$ y $15,45 \%$, en cada caso (tabla 2 ).

\section{Tipo 5: Tiempo aceptable con cobertura nubosa parcial}

Mantiene una distribución bimodal no acentuada, similar a la mostrada por el tipo 1 , si bien, a diferencia de este último, sus frecuencias resultan sensiblemente inferiores, cierto que relativamente elevadas. Asimismo, presenta un seno central más amplio, interrumpido por un pico (primera decena del mes de agosto, 14,33\%).

Su práctica ausencia equinoccial y, particularmente, invernal hay que relacionarla con la «escasa» radiación ligada a esta temperie y la poca eficacia calórica de ésta durante dicho período. Mientras que su proyección al final del equinoccio de primavera, e inicio del otoñal, así como, particularmente, su vocación estival (tabla 2), creemos que mantiene relación con: la frecuencia 
durante este periodo de tipos de tiempo marcados por la acumulación de nubosidad a favor de situaciones estables con flujos marítimos, la mayor parte de las veces de onda raigambre térmica; nubosidad producto de desarrollos termo-convectivos que tienen su origen en el interior del litoral mediterráneo de la península ibérica; y desarrollos nubosos ligados a situaciones mixtas (vaguada-cresta) (vid. Martínez, 2004).

\section{Tipo 6: Tiempo aceptable algo fresco soleado}

Muestra una distribución bimodal con presencia de picos máximos próximos al equinoccio de primavera y la llegada del solsticio de invierno. Este reparto anual no es sino producto de la horquilla térmica que lo delimita. Ciertamente, las frecuencias más significativas, del $24,33 \%$ y el $23 \%$, se han alcanzado durante la segunda decena del mes abril y la segunda decena del mes de noviembre, respectivamente.

Un matiz que dota de "personalidad» a esta clase de temperie es que mantiene unas frecuencias relativamente importantes durante el invierno (tabla 2).

\section{Tipo 7: Tiempo aceptable con breve episodio lluvioso}

Al igual que el tipo 5, esta clase de situaciones, más o menos inestables y sin apenas precipitación, únicamente se muestran relativamente presentes desde la segunda mitad del período primaveral hasta mediados de otoño, con un máximo de escasa significación, de 7,58\%, en la tercera decena del mes agosto (tabla 2).

\section{Tipo 8: Tiempo aceptable con episodio lluvioso}

Su frecuencia a lo largo del período anual resulta muy similar al de la clase anterior, analogía que no es reflejo sino de su parentesco.

No obstante, los valores porcentuales aún resultan de menor significación, hasta el extremo de presentar unos máximos francamente débiles, tal y como lo evidencia la cifra más importante alcanzada por este tipo, $2,73 \%$ en la tercera decena del mes de agosto (tabla 2).

Ciertamente, una mayor cantidad de precipitación, respecto al anterior (tipo 7), se relaciona, por norma general, con mayores índices de inestabilidad y, a la postre, menores fracciones de insolación, así como con unos registros térmicos inferiores. Factores que, en conjunto, determinan una disminución de la aptitud climático-turística de las jornadas con precipitaciones comprendidas entre 1 y $5 \mathrm{~mm}$. 
De hecho, entre los meses de mayo y octubre únicamente un $23 \%$ de los días con precipitación comprendida entre 1 y $5 \mathrm{~mm}$ se han considerado aceptables para la práctica turística. Las situaciones que han quedado fuera de este tipo, y que, por tanto, se han definido como inapropiadas para la práctica turística aquí analizada, se han relacionado, en un $77,20 \%$ de las jornadas, con fracciones de insolación inferiores al 50\%, y en un 68,39\% con registros térmicos que no han alcanzado la cifra de los $25^{\circ} \mathrm{C}$.

\section{Tipo 9: Tiempo aceptable con fuerte viento}

De nuevo, ésta es una situación de tiempo que muestra frecuencias muy escasas, dadas las coordenadas en las que se sitúa el observatorio analizado, así como el entorno geográfico en el que se localiza. Al respecto, los determinantes térmicos que lo definen explican la vocación estival del mismo (tabla 2). En este sentido, cabe traer a colación que, fuera del período más cálido, elevados registros térmicos en compañía de vientos relativamente importantes encuentran su explicación en episodios de foehn, con masas de aire de origen meridional.

\section{Tipo 10: Tiempo aceptable fresco soleado}

De entre los ambientes soleados, sin precipitación y vientos poco importantes, es el que muestra tanto una mayor predilección por el período invernal como un hiato estival más pronunciado. En efecto, al ser un tipo de tiempo relativamente fresco, la distribución de sus valores porcentuales no podría adquirir otra distribución anual. Así es, el carácter subtropical del sector suroriental de la península ibérica explica este hecho (tabla 2).

\section{Tipo 11: Tiempo aceptable relativamente fresco con cobertura nubosa parcial}

Como todas las variantes templadas presenta una distribución bimodal harto acentuada, con picos máximos durante los períodos equinocciales. Frecuencias significativas, pues, durante el mes de mayo y la primera decena de junio, y, en menor medida, en octubre y en la primera decena del mes de noviembre. Respecto a los valores porcentuales, cabe señalar que alcanzan cifras de al menos un 5\%, con un máximo de un $8,18 \%$ en la tercera decena del mes de mayo (tabla 2). 
Tipo 12: Tiempo aceptable relativamente fresco con breve episodio lluvioso

Se caracteriza, principalmente, por su exigua frecuencia de aparición, con máximos equinocciales de escasa significación, de 1,67\% en la primera decena del mes de mayo y un $3 \%$ en la segunda decena del mes de octubre (tabla 2).

\section{Tipo 13: Tiempo aceptable relativamente fresco con fuerte viento}

Su reparto porcentual decenal a lo largo del año aparece inverso a la variable más cálida de este tipo, presentando, pues, una distribución de frecuencias bimodal.

Asimismo, si ya el tipo más cálido se caracterizaba por su escasa manifestación, en este caso, ésta aún se muestra menos significativa; a la postre, esta clase de tiempo es, tal y como ya se ha indicado, la de menor proyección. De este modo, los máximos decenales apenas si rebasan el valor del $1 \%$, en la primera decena del mes de mayo y durante la segunda del mes de octubre (tabla 2).

\section{Determinación de la temporada climático-turística para la práctica recreativa del turismo de sol y playa en Alicante}

En primer lugar, conviene tener presente que se toman en consideración los umbrales planteados por B. Gómez (2000: 350). En este sentido, la autora considera que frecuencias decenales de tipos de tiempo aptos entre el 50\% y el $60 \%$ contentarían a aquellos turistas con pretensiones más bajas; valores entre el $60 \%$ y el $70 \%$ harían lo propio con grados de exigencia medios; mientras que porcentajes de al menos un $70 \%$ satisfarían a aquellos turistas más exigentes.

Aplicando los mismos criterios a la clasificación aquí expresada, los límites del período que hipotéticamente complacería a la clientela «más flexible» se situarían entre la primera decena del mes de abril y la última de octubre. Bien es cierto que, los valores alcanzados entre la segunda decena del mes marzo y su homóloga del mes de noviembre quedan próximos a estas hipotéticas pretensiones.

Desde la segunda decena del mes de mayo hasta la tercera del mes de octubre las frecuencias de tiempo aceptable para la práctica del turismo de sol y playa quedan por encima del valor del $60 \%$, colmando pues, en teoría, las necesidades de aquellos turistas con exigencias moderadas.

Mientras que valores de al menos un $70 \%$ se han alcanzado desde la segunda decena de mayo hasta la primera del mes de octubre. 
Tabla 3. Evaluación del potencial climático-turístico decenal y su variabilidad para el turismo de sol y playa en Alicante (1974-2003)

\begin{tabular}{|c|c|c|c|c|c|c|}
\hline Mes & Decena & $\begin{array}{c}\text { Aceptables } \\
(\%)\end{array}$ & $\begin{array}{l}\text { Frecuencia } \\
\text { máxima }\end{array}$ & $\begin{array}{l}\text { Frecuencia } \\
\text { mínima }\end{array}$ & Recorrido & $\mathrm{CV}$ \\
\hline E & 1 & 22,00 & 7 & 0 & 7 & 142,47 \\
\hline E & 2 & 18,33 & 7 & 0 & 7 & 173,73 \\
\hline E & 3 & 20,91 & 6 & 0 & 6 & 138,43 \\
\hline $\mathrm{F}$ & 1 & 34,67 & 10 & 0 & 10 & 91,82 \\
\hline $\mathrm{F}$ & 2 & 26,00 & 9 & 0 & 9 & 122,61 \\
\hline $\mathrm{F}$ & 3 & 25,52 & 6 & 0 & 6 & 151,58 \\
\hline $\mathrm{M}$ & 1 & 36,00 & 8 & 0 & 8 & 88,36 \\
\hline$M$ & 2 & 41,33 & 8 & 0 & 8 & 77,10 \\
\hline $\mathrm{M}$ & 3 & 45,15 & 10 & 0 & 10 & 64,15 \\
\hline $\mathrm{A}$ & 1 & 50,00 & 10 & 1 & 9 & 63,70 \\
\hline $\mathrm{A}$ & 2 & 51,67 & 10 & 1 & 9 & 61,67 \\
\hline $\mathrm{A}$ & 3 & 46,67 & 9 & 1 & 8 & 68,26 \\
\hline $\mathrm{M}$ & 1 & 56,00 & 9 & 2 & 7 & 56,85 \\
\hline $\mathrm{M}$ & 2 & 72,00 & 10 & 1 & 9 & 44,21 \\
\hline $\mathrm{M}$ & 3 & 71,21 & 11 & 2 & 9 & 40,67 \\
\hline $\mathrm{J}$ & 1 & 78,67 & 10 & 5 & 5 & 40,50 \\
\hline $\mathrm{J}$ & 2 & 85,33 & 10 & 4 & 6 & 37,32 \\
\hline $\mathrm{J}$ & 3 & 84,67 & 10 & 3 & 7 & 37,61 \\
\hline $\mathrm{J}$ & 1 & 83,67 & 10 & 5 & 5 & 38,04 \\
\hline $\mathrm{J}$ & 2 & 84,33 & 10 & 5 & 5 & 37,73 \\
\hline $\mathrm{J}$ & 3 & 83,64 & 11 & 6 & 5 & 34,57 \\
\hline $\mathrm{A}$ & 1 & 74,00 & 10 & 0 & 10 & 42,98 \\
\hline $\mathrm{A}$ & 2 & 76,67 & 10 & 1 & 9 & 41,50 \\
\hline $\mathrm{A}$ & 3 & 71,21 & 11 & 1 & 10 & 40,60 \\
\hline$S$ & 1 & 83,00 & 10 & 5 & 5 & 38,31 \\
\hline$S$ & 2 & 85,00 & 10 & 5 & 5 & 37,42 \\
\hline$S$ & 3 & 78,67 & 10 & 4 & 6 & 40,43 \\
\hline $\mathrm{O}$ & 1 & 78,33 & 10 & 4 & 6 & 40,59 \\
\hline $\mathrm{O}$ & 2 & 67,00 & 10 & 3 & 7 & 47,43 \\
\hline $\mathrm{O}$ & 3 & 64,85 & 11 & 4 & 7 & 44,53 \\
\hline $\mathrm{N}$ & 1 & 49,00 & 9 & 1 & 8 & 64,81 \\
\hline $\mathrm{N}$ & 2 & 46,67 & 9 & 0 & 9 & 68,05 \\
\hline $\mathrm{N}$ & 3 & 36,00 & 9 & 0 & 9 & 88,18 \\
\hline $\mathrm{D}$ & 1 & 28,33 & 8 & 0 & 8 & 112,09 \\
\hline $\mathrm{D}$ & 2 & 28,00 & 6 & 1 & 5 & 113,41 \\
\hline $\mathrm{D}$ & 3 & 17,88 & 9 & 0 & 9 & 161,41 \\
\hline
\end{tabular}

Fuente: AEMET. Elaboración propia. 
Tabla 4. Tipos de tiempo definidos (incluyendo la clase desfavorable) para la práctica del turismo de sol y playa en el litoral de Alicante reducidos a una escala valorativa de 0 a 10

\begin{tabular}{|c|c|c|c|}
\hline Tipo de tiempo & Valor asignado & Tipo de tiempo & Valor asignado \\
\hline 1 & 10,00 & 8 & 7,08 \\
\hline 2 & 9,58 & 9 & 6,66 \\
\hline 3 & 9,17 & 10 & 6,25 \\
\hline 4 & 8,75 & 11 & 5,83 \\
\hline 5 & 8,33 & 12 & 5,41 \\
\hline 6 & 7,92 & 13 & 5,00 \\
\hline 7 & 7,50 & Desfavorable & 0,00 \\
\hline
\end{tabular}

Fuente: Elaboración propia.

Toda vez que se ha realizado un seguimiento temporal, a resolución decenal, de los tipos de tiempo favorables y desfavorables, detallando asimismo la distribución de las frecuencias de cada uno de ellos, se ha creído que pudiera resultar conveniente trasladar a una escala de valores dichos resultados.

Figura 1. Valoración, en una escala de 0 a 10, de las decenas anuales para la práctica del turismo de sol y playa en Alicante (1974-2003)

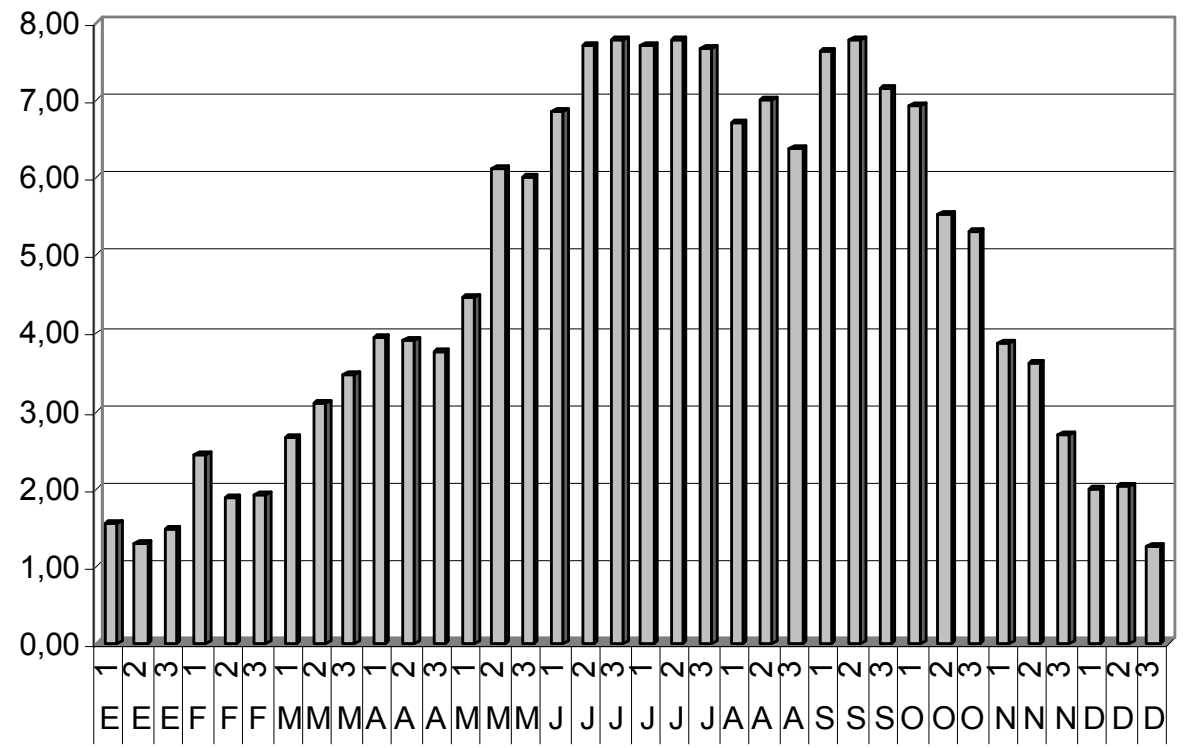

Fuente: AEMET. Elaboración propia. 
Figura 2. Reparto mensual de aquellos tipos de tiempo definidos como no aptos para la práctica turística por estrés térmico positivo en Alicante (1974-2003)

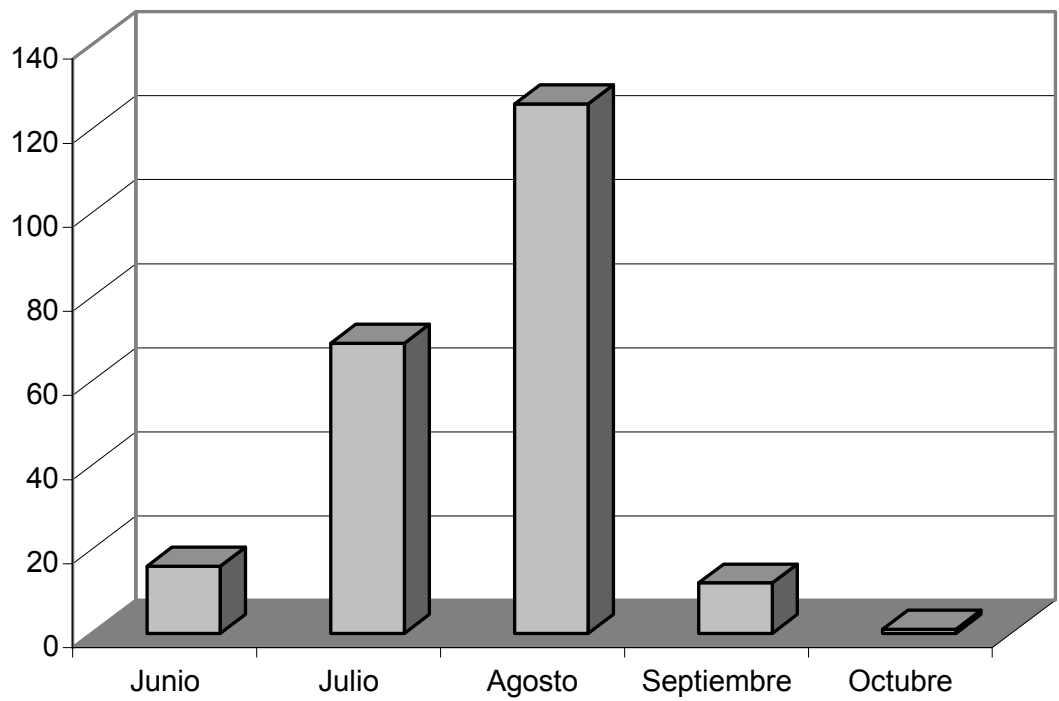

Fuente: AEMET. Elaboración propia.

Una primera aproximación que, si bien no está exenta de significación, debe ser refinada a partir de encuestas sobre tiempo percibido, creemos que puede ser abordada de la siguiente forma: reducir los 13 tipos definidos como aceptables según su orden de aptitud climático-turística a una escala de valores de 5 a 10. La primera cifra se corresponde con el último tipo clasificado como tolerable, es decir, Tiempo aceptable relativamente fresco con fuerte viento, mientras que el valor máximo representa una aptitud ideal, tipo de tiempo 1, esto es, Tiempo muy bueno soleado. De esta forma, la escala para cada clase de tiempo definida queda tal y como aparece en la tabla 4 (las situaciones desfavorables obtendrían la puntuación de 0). Aplicando dicha equivalencia se alcanzaría el potencial decenal anual reflejado en la figura 1.

Según esta propuesta, se observa cómo el período que queda por encima de los cincos puntos comprende desde la segunda decena del mes de mayo hasta la tercera del mes de octubre ${ }^{5}$, ambas incluidas. Margen temporal que

5. Téngase en cuenta que Palomares (1965) consideró que el tiempo «estival», aquel en el que las temperaturas medias de las máximas superan los $25^{\circ} \mathrm{C}$, se extiende desde mayo a mediados de octubre en el litoral levantino y la Costa del Sol (vid. J. del Valle Melendo, 2007). 
coincide con valores de al menos un 60\% de probabilidad empírica de disfrutar de tiempo aceptable para la práctica turística. Por ello, se cree apropiado definir la temporada de sol y playa entre ambos límites.

De otro lado, conviene tener presente que ya desde la primera decena del mes de abril y hasta la segunda del mes de noviembre la valoración de los ambientes atmosféricos se aproxima al valor cuatro. Como quiera que estos umbrales temporales resultan similares a los considerados para una clientela menos exigente, se cree que la temporada de bronceado puede quedar entre las mencionadas decenas.

Además de lo hasta ahora indicado, cabe subrayar dos aspectos. De un lado, la relación inversa que se establece entre potencial climático-turístico y variabilidad del mismo; es decir, a superior aptitud corresponde mayor seguridad de disfrutar de condiciones meteorológicas aceptables para la práctica

Figura 3. Frecuencia de días con lluvia (\% respecto al total de jornadas) para cada período decenal en Alicante (1974-2003)

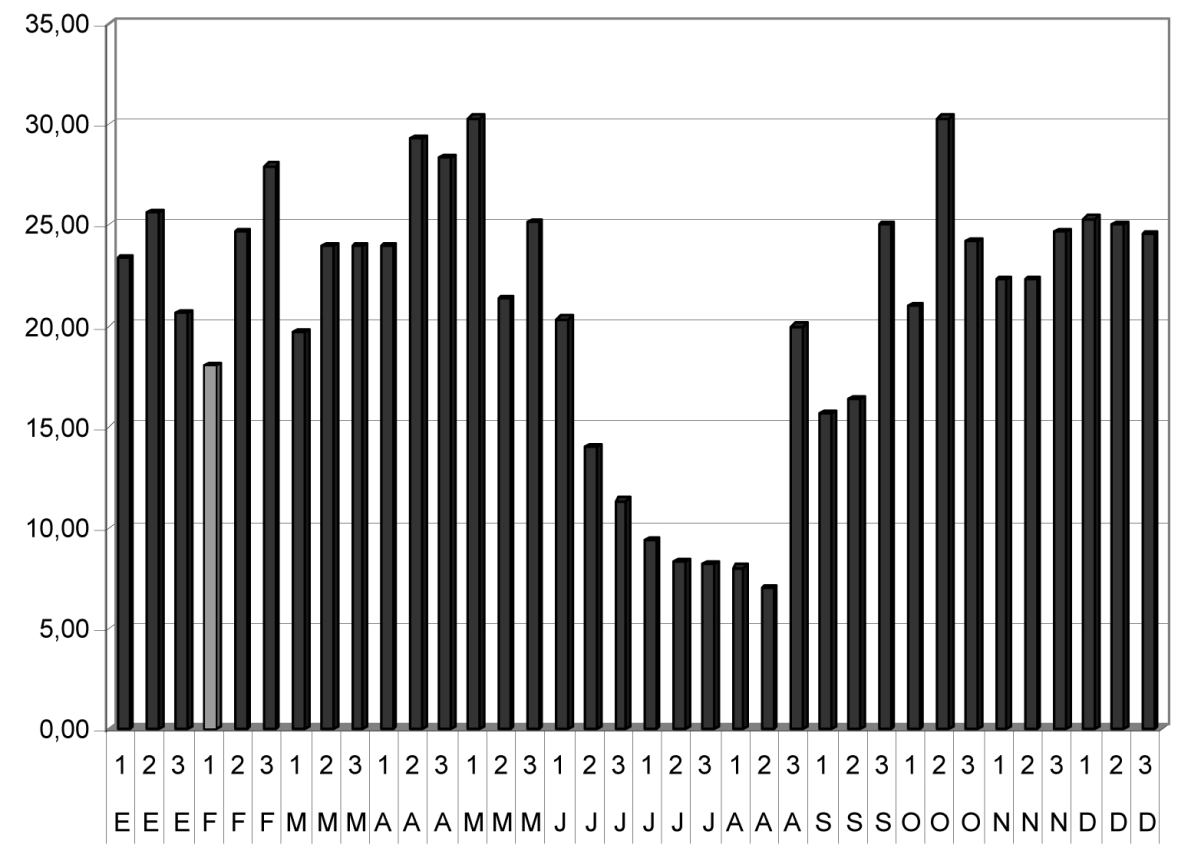

Fuente: AEMET. Elaboración propia. 
Figura 4. Número de jornadas con precipitación comprendida entre 0 y 1 mm. en Alicante (1974-2003)

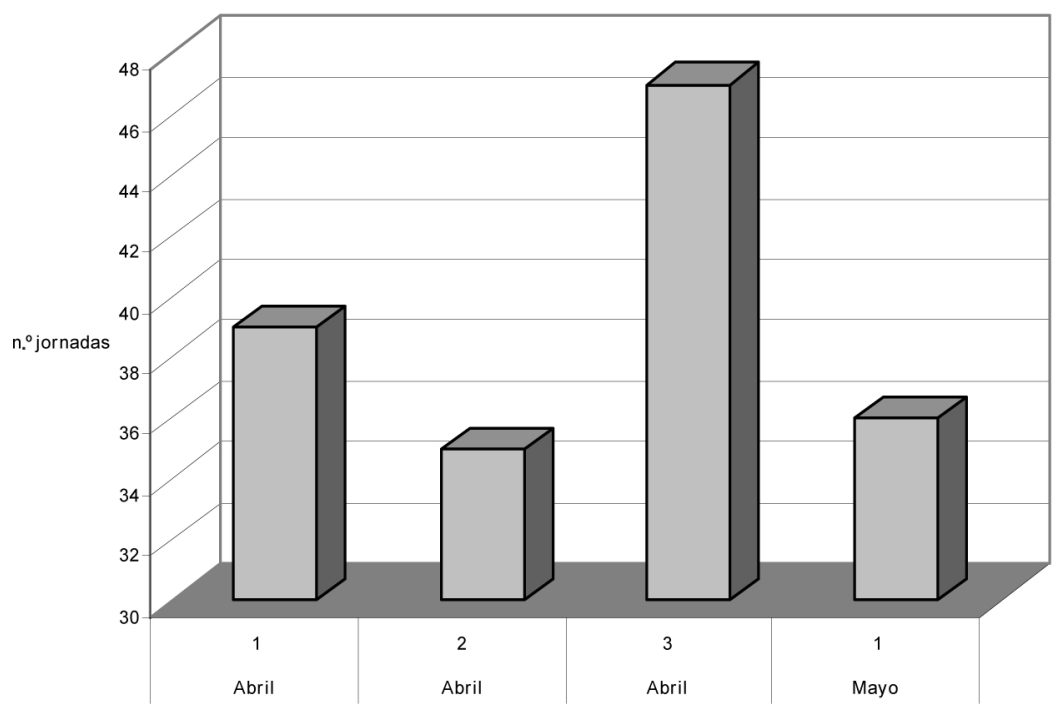

Fuente: AEMET. Elaboración propia.

turística ${ }^{6}$. De otro, el seno que se observa durante el mes de agosto. Tendencia ésta opuesta a la realidad de los flujos turísticos. Este hecho ha condicionado asimismo la existencia de mínimos decenales de 0 y 1 durante esta mensualidad (tabla 3).

Asimismo, con relación a la evolución mensual del potencial climáticoturístico, merece la pena aludir a dos tendencias contrarias. Por un lado, destaca el incremento del potencial (en términos de frecuencia y seguridad) registrado durante la primera decena de febrero, y, de otro, el declive que se produce en la tercera decena del mes de abril. Por lo que concierne al primer aspecto, las causas que pueden explicar dicha evolución pueden encontrar razón de ser en la escasa trascendencia de días con lluvia durante ese período, ligada quizá al período de calma que acontece durante el centro del invierno (obsérvese la columna gris de la figura 3). Por su parte, con relación a la segunda tendencia, ésta parece mostrar relación con el incremento de las situaciones inseguras durante abril, particularmente en su segunda mitad. Así, durante el período analizado, se han registrado tanto fracciones de insolación

6. Aspecto también indicado por Gómez (2000) para el caso de Cataluña. 
inferiores como, especialmente, una mayor frecuencia de días con lluvia poco importante (figura 4). De hecho, J.P. Besancenot (1985: 443) para las costas Ibéricas señala que el principal inconveniente del período primaveral es su elevada irregularidad, especialmente la interanual ${ }^{7}$. De esta forma, aunque los datos medios parecen dotar a esta estación de un atractivo suficiente, la irregularidad implica para el turista un riesgo significativo de ver frustradas sus vacaciones (J.P. Besancenot, 1985: 443).

\section{Conclusiones}

Para el conjunto del período anuo, el principal aspecto a subrayar es el predominio de las condiciones favorables para la práctica del turismo de sol y playa. En este sentido, conviene asimismo tener presente la primacía de las clases de tiempo más aptas, los tipos de tiempo cálidos $(1,2$ y 3$)$.

Otro aspecto que llama poderosamente la atención es el seno que se manifiesta en las frecuencias de aptitud climático-turística con ocasión del mes de agosto, con mínimos decenales que han alcanzado valores realmente ínfimos.

Por último, conviene hacer notar que la temporada de bronceado en Alicante puede quedar delimitada entre la primera decena del mes de abril y la segunda del mes de noviembre; mientras que la de bronceado y baño parece quedar comprendida entre la segunda decena del mes de mayo y la tercera del mes de octubre.

\section{Bibliografía}

Andrade, H., M.J. Alcoforado y S. Oliveira (2007): «How does climate effectively affect tourism? The advantage of weather type methodology», en A. Matzarakis, C.R. De Freitas, y D. Scott (eds.): $3^{\text {rd }}$ Internacional workshop on climate, tourism and recreation, International Society of Biometeorology, Commission on Climate, Tourism and Recreation, Alexandroupolis, Grecia.

BAtista TAMAYO, L.M. y F. MATOS PUPO (2004): «La aptitud climática del destino turístico Jardines del Rey (Cuba). Los tipos de tiempo», en J.C. GARCía CoDRON et al. (eds.), Santander, 561-570.

BESANCENOT J.P., J. MOUNIER y F. De LAVENNE (1978): «Les conditions climatiques du tourisme littoral: une méthode de recherche compréhensive», en Norois, tomo XXV, n. ${ }^{\circ} 99$, pp. 357-382.

7. «[...] ningún otro periodo ve en las medias de la realidad una imagen tan infiel [...]» (J.P. Besancenot, 1985). 
BESANCENOT, J.P. (1985): «Climat et tourisme estival sur les côtes de la péninsule ibérique», en Revue Géographique des Pyrénées et du Sud-Ouest, tomo 56, fasc. 4, pp. 427-451.

- (1991): Clima y turismo, Massom, Barcelona.

Cuadrat Prats, J.M. (1983): «Método de clasificación de tipos de tiempo aplicados al turismo de montaña», en VIII Coloquio de geógrafos españoles, Barcelona, pp. 11-16.

Chávez Del Valle, FJ. (2002): Zona variable de confort térmico, tesis doctoral inédita, Barcelona, 158 pp.

Dauphiné, A. y N. Ghilardi (1978): «Essai de bioclimatologie touristique: la Cote d'Azur», en Méditerraneé (Revue Géographique des Pays Méditerranéens), tomo 33, no 3, pp. 3-15.

DE FREITAS, C.R. (1990): «Recreation climate assessment», en International Journal of Climatology, vol. 10, pp. 89-103.

- (2001): «Theory, concepts and methods in tourism climate research», en A. Matzarakis, A. y C.R. DE Freitas (eds.): Proceedings of the First International workshop on climate, tourism and recreation, International Society of Biometeorology, Commission on Climate, Tourism and Recreation, Porto Carras, Halkidiki, Grecia.

- (2003): «Tourism climatology: evaluating environmental information for decision making and business planning in the recreation and tourism sector». International Journal of Biometeorology, n. ${ }^{\circ} 48$, pp. 45-54.

- (2005): «The climate-tourism relationship and its relevance to climate change impact assessment», en C.M. HALl y J. Higham, (eds.): Tourism, recreation and climate change, Channel View Publications, Clevelon-Buffalo-Toronto.

- D. Scott y G. Mcboyle (2004): «A new generation climate index for tourism», en A. Matzarakis, C.R. De Freitas, y D. Scott (eds.): Advances in tourism climatology, Friburgo, pp. 19-26.

Del Valle Melendo, J. (2007): Turismo y clima: un diálogo necesario, en Estudios Turísticos, n. ${ }^{\circ} 171$, pp. 77-91.

GÓMEz Martín, B. (2000): Clima y turismo en Cataluña: Evaluación del potencial climático-turístico de la estación estival, tesis doctoral, Universidad de Barcelona, Barcelona, 551 pp.

- (2003): «Duración y características de la estación climático-turística estival en Cataluña, en Estudios Geográficos, LXIV, n. ${ }^{\circ}$ 253, pp. 623-653.

MARTínez IBARra, E. (2004): «Aportaciones para el análisis de un clima local: variación estacional de los estados del cielo en Benidorm», en Investigaciones Geográficas, n. ${ }^{\circ}$ 35, pp. 133-150.

- (2008): Tipos de tiempo para el turismo de sol y playa en el litoral alicantino, Estudios Geográficos, LXIX, n. ${ }^{\circ}$ 264, pp. 133-155. 
MATZARAKIS, A. (2007): «Assessment method for climate and tourism based on daily data», en A. MAtzarakis,, C.R. DE Freitas, y D. Scott (eds.) (2007): $3^{\text {rd }}$ Internacional workshop on climate, tourism and recreation, International Society of Biometeorology, Commission on Climate, Tourism and Recreation, Alexandroupolis, Grecia.

Scout, D., S. GÖsSling y C.R. DE Freitas (2007): «Climate preferences for tourism: An exploratory tri-nation comparison», en A. MATZARAKIS, C.R. DE FREITAS y D. Scott (eds.): $3^{\text {rd }}$ Internacional workshop on climate, tourism and recreation, International Society of Biometeorology, Commission on Climate, Tourism and Recreation, Alexandroupolis, Grecia. 